\title{
HIGHLIGHTS
}

CANCER

\section{Oral bisphosphonates could raise risk of esophageal cancer}

Long-term use of oral bisphosphonates might increase the risk of esophageal cancer, according to a UK study. The risk was also affected by the number of prescriptions for a bisphosphonate that the patient received.

Patients who take oral bisphosphonates to prevent or treat osteoporosis often have adverse gastrointestinal events, such as dyspepsia, nausea, esophageal ulcers and erosive esophagitis. "We were already interested in the long-term effects of use of bisphosphonates, and were spurred on by the publication of FDA case reports of esophageal cancer in bisphosphonate users," explains lead author Jane Green (University of Oxford, UK).

As esophageal cancer is not common, the researchers used a case-control approach to analyze the General Practice Research Database-a record of the health care received by 6 million people in the UK. Data from 2,954 patients with esophageal cancer, 2,018 with stomach cancer, 10,641 with colorectal cancer and 77,750 matched controls was analyzed over a mean follow-up period of 7.5 years. Around 3\% of the patients included in the analysis had at least one prescription for an oral bisphosphonate.

Green and colleagues found that taking oral bisphosphonates for $\geq 5$ years doubled the risk of developing esophageal cancer. The number of prescriptions a patient received also influenced their risk; $\geq 10$ prescriptions significantly raised the risk compared to 1-9 prescriptions. The type of bisphosphonate administered did not effect the risk of esophageal cancer. Use of bisphosphonates did not alter the risk of stomach or colorectal cancer.

Very little work has been published on the effects of long-term use of oral bisphosphonates. One other study, which also analyzed data from the General Practice Research Database, found no evidence of an increased risk of esophageal cancer in patients who use bisphosphonates. This lack of consensus suggests that more research needs to be done to assess the adverse effects of the long-term use of these drugs, particularly as their use is increasing (3\% of women $>70$ years in the UK received a prescription in 2000, compared with $10 \%$ in 2005). Indeed, Green suggests that their analysis needs to be repeated in the future with a longer follow-up period.

"Esophageal cancer is uncommon and even if our findings are confirmed the absolute risk in bisphosphonate users will remain low," concludes Green. "We would like to stress the need for-and current lack of-reliable information on all the benefits and risks of long-term use of these drugs."

Claire Greenhill

Original article Green, J. et al. Oral bisphosphonates and
risk of cancer of oesophagus, stomach, and colorectum:
case-control analysis within a UK primary care cohort. BMJ
341, c4444 (2010)

